\title{
Monitoring of systemic candidiasis by 18F-FDG PET/CT
}

\author{
John Avet Jr. • Denise Granjon • Nathalie Prevot-Bitot • \\ Vanina Isnardi • Claire Berger • Jean Louis Stephan • \\ Francis Dubois
}

Received: 29 April 2009 / Accepted: 31 July 2009 /Published online: 1 September 2009

(C) Springer-Verlag 2009

A 16-year-old patient was treated for leukemia and after chemotherapy developed fever, myalgia, myocardial dysfunction, and athralgia. Blood culture showed Candida tropicalis. Contrast-enhanced computed tomography showed multiple kidney and spleen abscesses. After antifungal therapy (TRIFLUCAN) and before restarting chemotherapy, ${ }^{18}$ F-FDG PET/CT was performed [1], which demonstrated multiple high metabolic activity lesions in the spleen and kidneys, and unknown disseminated lesions in muscles, myocardium, the right lung, and liver [2,3]. Bonemarrow-increased metabolic activity seems to be related to the recent chemotherapy treatment. Clinical and ${ }^{18} \mathrm{~F}-\mathrm{FDG}$ PET/CT evolution was successful after a new antifungal therapy (NOXAFIL).

This case illustrates the usefulness of ${ }^{18} \mathrm{~F}$-FDG PET/CT
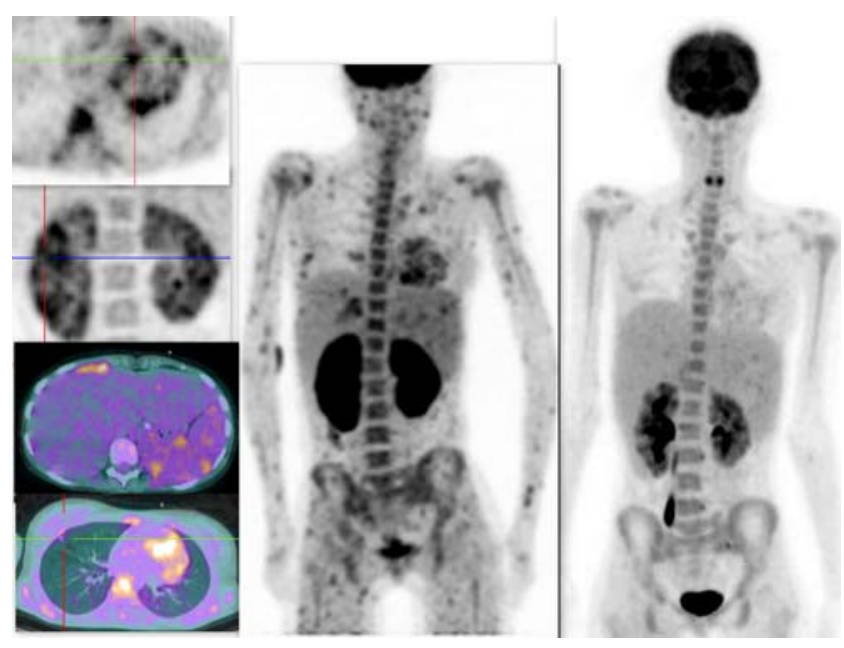
in the identification of disseminated candidiasis sites and in the evaluation of antifungal treatment [4].

J. Avet Jr. $(\bowtie) \cdot$ D. Granjon $\cdot$ N. Prevot-Bitot $\cdot$ V. Isnardi $\cdot$

F. Dubois

Department of Nuclear Medicine,

CHU de Saint Etienne, France

e-mail: johnavet@free.fr

C. Berger $\cdot$ J. Louis Stephan

Department of Paediatric Oncology,

CHU de Saint Etienne, France

\section{References}

1. O'Doherty MJ, Barrington SF, Klein JL. Opportunistic infection and nuclear medicine. Semin Nucl Med. 2009;39(2):88-102.

2. Bleeker-Rovers CP, Vos FJ, Wanten GJ, van der Meer JW, Corstens $\mathrm{FH}$, Kullberg BJ, et al. FDG PET in detecting metastatic infectious disease. J Nucl Med. 2005;46(12):2014-9.

3. Zhuang H, Yu JQ, Alavi A. Applications of FDG PET imaging in detection of infection and inflammation and other benign disorders. Radiol Clin North Am. 2005;43(1):121-34.

4. Bleeker-Rovers CP, Warris A, Drenth JP, Cortens FH, Oven WJ, Kullberg BJ. Diagnosis of Candida lung abscesses by $18 \mathrm{~F}-$ fluorodeoxyglucose positron emission tomography. Clin Microbiol Infect. 2005;11(6):493-5. 